\title{
Optical signal impairment study of cascaded filters in 40 Gbps DQPSK and 100 Gbps PM-DQPSK systems
}

\author{
Xiaoyong Chen $*^{\mathrm{a}}$, Paloma R. Horche ${ }^{\mathrm{a}}$, Alfredo M. Minguez ${ }^{\mathrm{b}}$

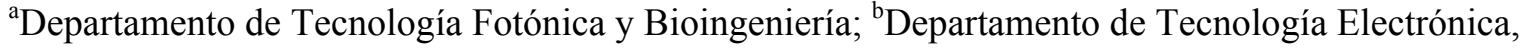 \\ ETSI Telecomunicación, Universidad Politécnica de Madrid, Avda. Complutense 30, Madrid, Spain 28040
}

\begin{abstract}
Optical filters are crucial elements in optical communications. The influence of cascaded filters in the optical signal will affect the communications quality seriously. In this paper we will study and simulate the optical signal impairment caused by different kinds of filters which include Butterworth, Bessel, Fiber Bragg Grating (FBG) and Fabry-Perot (FP). Optical signal impairment is analyzed from an Eye Opening Penalty (EOP) and optical spectrum point of view. The simulation results show that when the center frequency of all filters aligns with the laser's frequency, the Butterworth has the smallest influence to the signal while the F-P has the biggest. With a $-1 \mathrm{~dB}$ EOP, the amount of cascaded Butterworth optical filters with a bandwidth of $50 \mathrm{GHz}$ is 18 in $40 \mathrm{Gbps}$ NRZ-DQPSK systems and 12 in $100 \mathrm{Gbps}$ PMNRZ-DQPSK systems. The value is reduced to 9 and 6 respectively for Febry-Perot optical filters. In the situation of frequency misalignment, the impairment caused by filters is more serious. Our research shows that with a frequency deviation of $5 \mathrm{GHz}$, only 12 and 9 Butterworth optical filters can be cascaded in 40 Gbps NRZ-DQPSK and 100 Gbps PM-NRZ-DQPSK systems respectively. We also study the signal impairment caused by different orders of the Butterworth filter model. Our study shows that although the higher-order has a smaller clipping effect in the transmission spectrum, it will introduce a more serious phase ripple which seriously affects the signal. Simulation result shows that the 2nd order Butterworth filter has the best performance.
\end{abstract}

Keywords: optical filter, optical communication, differential quadrature phase shift keying (DQPSK), polarization multiplexed differential quadrature phase shift keying (PM-DQPSK), signal impairment

\section{INTRODUCTION}

Optical filters are very important elements in optical networks, especially in long-haul optical communications. In the transparent optical network, an optical signal may be passing through several optical cross-connects (OXCs) nodes, Dispersion Compensation Modules (DCMs) which are based on chirped fiber Bragg gratings, and reconfigurable optical add-drop multiplexers (ROADMs) before it reaches the destination $[1,2]$. Normally, the OXCs and ROADMs comprise of several filters, such as wavelength blockers (WB) and wavelength selective switches (WSS). When the signal passes through several cascaded OXCs or ROADMs, its influence toward the signal can be referred to as the filters concatenation effect [3]. This effect impacts the signal quality seriously since that cascaded filters make the filter passband narrow which will lead to signal spectrum clipping effect, and introduce strong chromatic dispersion because of the variations in filter phase ripple, loss ripple and group delay ripple [4]. With the amount of cascaded filters increasing, the filter concatenation effect will be more severe and the signal quality will be further aggravated. Furthermore, when the center frequency of the filter passband does not align to the optical signal frequency, such effect will be amplified and cause more serious impairment to the signal. In order to keep the signal transmission with high quality in the long-haul communication network, it is very significant to study the signal impairment caused by different kinds of cascaded filters.

*xiaoyong.chen@alumnos.upm.es; phone: 3491 336-3408; fax: 3491 336-7319; www.tfo.upm.es

Optics and Photonics for Information Processing VII, edited by Khan M. Iftekharuddin,

Abdul A. S. Awwal, Andrés Márquez, Proc. of SPIE Vol. 8855, 88550C · (C) 2013 SPIE

CCC code: $0277-786 X / 13 / \$ 18 \cdot$ doi: $10.1117 / 12.2021521$

Proc. of SPIE Vol. $885588550 \mathrm{C}-1$ 
Many papers have studied the effects of filter concatenation in optical communication. In [5] the performance of a cascade of 11 filters with bandwidth of $50 \mathrm{GHz}$ has been studied in a $20 \mathrm{~Gb} / \mathrm{s}$ NRZ system. In $[3,4,6]$ signal impairment caused by filter concatenation in coherent optical OFDM system has been presented. Simulation of signal impairment caused by cascaded ROADMs has been shown in [7]. Signal distortion and crosstalk penalties induced by optical filters in optical networks have been commented in [8]. Analysis of loss ripple, group delay ripple and dispersion induced by optical filters have presented in $[9,10,11]$. However, almost all the papers mentioned above just discuss the performance of one kind of filters. In optical communication system, there are many kinds of optical filter models, such as Gaussian optical filter, Butterworth optical fiber, Bessel optical filter, fiber Bragg grating (FBG), Fabry-Perot optical filter and so on. As demonstrated in this paper, different filters have different performance and the filter amount of permitted to be cascaded in the system is different. Besides, different orders of the filter also have different performance. In [7] performance of different orders of Gaussian filter has been discussed. In this paper we will study and simulate the optical signal impairment caused by different kinds of filters which include Butterworth filter, Bessel filter, Fiber Bragg Grating (FBG) and Fabry-Perot filter. Optical signal impairments in 40 Gbps DQPSK and 100 Gbps PM-DQPSK systems are analyzed from eye opening penalty (EOP), eye diagrams and optical spectrum. Also, different effects caused by different orders of Butterworth filter are discussed from both transmission function and transmission phase. The paper is organized as follows: In Section 2 we will present the characteristic of optical filters concatenation; In Section 3 we will describe the transmission scheme and the simulation design; then the simulation results will be presented and discussed in Section 4; finally, the conclusion is given in Section 5.

\section{CHARACTERISTICS OF CASCADED OPTICAL FILTERS}

As mentioned before, in a Wavelength Division Multiplexing (WDM) optical network, before reaching the destination an optical signal may be filtered several times by passing through many optical filters, such as ROAMDs, DCMs and OXCs. Those filters will cause passband curvature tilt and ripple in the frequency response since they don't have ideal transfer functions for both optical amplitude and phase. Moreover, the phase transfer function cannot vary linearly with frequency. With the amount of cascaded filters increasing, it will increase the effect of spectral clipping and make the effective bandwidth narrower and narrower. This effect can lead to the signal distortion in the time domain, causing a Qfactor penalty or EOP $[2,8,12]$.

The effective transfer function of the cascaded filters is the product of every individual filter. Therefore, the effective transmission bandwidth of cascaded filters is much narrower than that of an individual filter. Moreover, when the center frequency of the cascaded filters is misalignment, it will further narrow the effective bandwidth. This effect can be seen from fig. 1. Compared with an individual 3rd order Butterworth filter, the effective bandwidth of 10 cascaded Butterworth filters is much narrow (red line), even if these 10 filters are aligned in the center frequency. When these 10 filters are cascaded with center frequency misalignment of $\pm 5 \mathrm{GHz}$, the effective bandwidth is further narrowed. This can be seen in the green line of fig. 1. That is because the transfer function of the filter is not an ideal rectangle transfer function. With the number of cascaded filters increasing, the flat top region the effective transfer function becomes smaller and the tail decay becomes steeper. This makes the effective bandwidth smaller, especially when the cascaded filters are not aligned perfectly.

According to [2], many filters have characteristics that are well approximated by a 3rd order Butterworth filter transfer function, such as the thin film filters that are widely used in WDM systems. In this paper we will focus on the 3rd Butterworth filter and compared its performance with Bessel, FBG and F-P filters. Besides, according to [15], an F-P filter can be approximated as the 1st order Butterworth filter model. Also, the 2nd and 4th order Butterworth filter model are studied in [16]. So in this part firstly we will study the Butterworth filter model with different orders.

Proc. of SPIE Vol. $885588550 \mathrm{C}-2$ 
Transmission Function

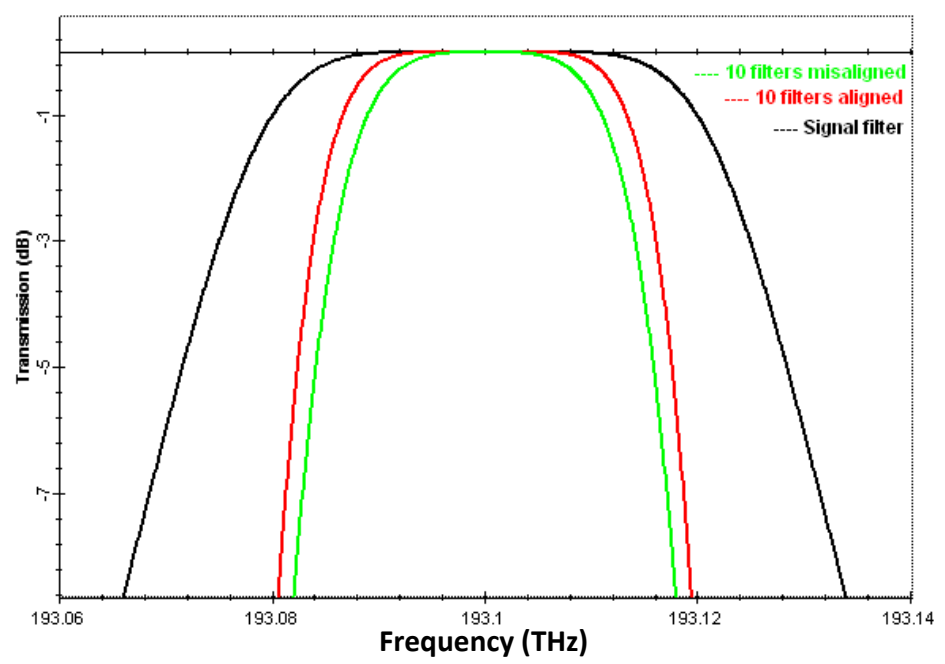

Figure 1. Effective transfer functions of a single 3rd Butterworth filter and 10 cascaded filters aligned/misaligned

\subsection{Butterworth filter}

According to [13], an $\mathrm{n}^{\text {th }}$ order Butterworth filter transfer function can be described as the following:

$$
H(f)=\frac{\alpha(B / 2)^{n}}{\prod_{k=0}^{n-1}\left(j\left(f-f_{C}\right)-\frac{B}{2} \cdot \exp \left(j \frac{\pi}{2}\left(1+\frac{2 k+1}{n}\right)\right)\right)}
$$

where $\alpha$ is the parameter Insertion loss, $f_{c}$ is the filter center frequency, B is the Bandwidth, $n$ is the order, and $f$ is the frequency. The characteristic of filter is decided by amplitude transfer function and phase transfer function. According to the Eq. (1), we can calculate the effective transfer function of various orders in the situations of individual and cascaded situation. The results are shown in fig. 2 and fig. 3.

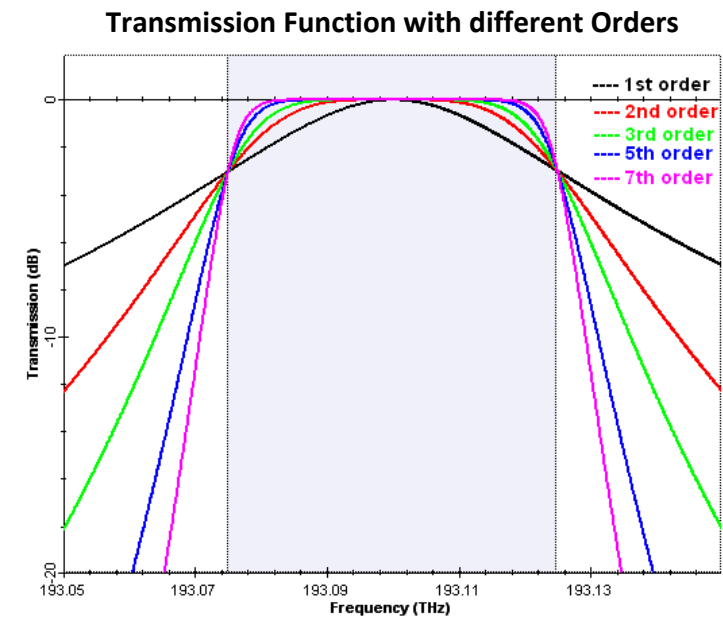

(a)

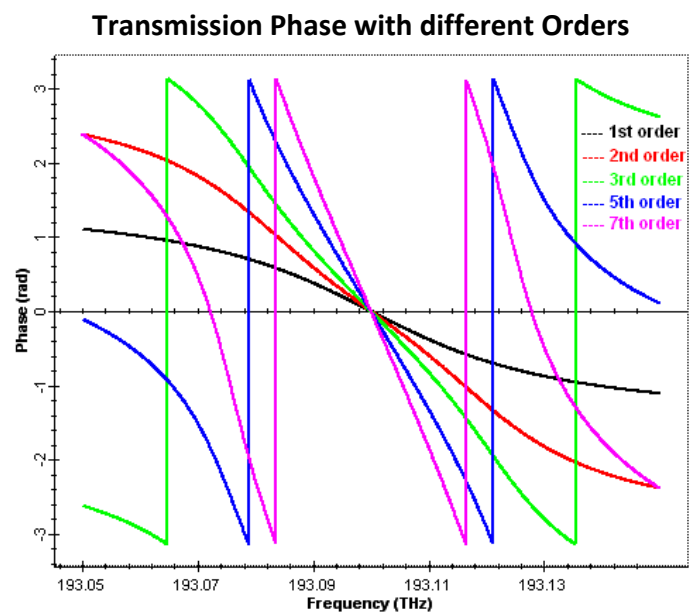

(b)

Figure 2. (a) Transmission amplitude of different orders of Butterworth filter model (Single filter); (b) Transmission phase of different orders Butterworth filter (Single filter) 
Fig. 2 shows the amplitude transfer function and phase transfer function of various orders of an individual Butterworth filter. We can see that with order increasing, the amplitude transfer function of Butterworth filter is much closer to the ideal rectangle transfer function. In other words, when the bandwidth is fixed, with the order increasing the flat top region is wider and the tail decay is steeper. However, the phase transfer function shows the opposite trend. With the order increasing, the linear region becomes smaller and the frequency dependence becomes more serious. Fig. $3 \mathrm{a}$ shows the effective bandwidth changing with the number of cascaded filters. It can be seen that the effective bandwidth becomes smaller with the filters increasing. The high-order shows less effect to the effective bandwidth. For example, the effective bandwidth of 30 cascaded 1st order filters $(50 \mathrm{GHz})$ is just $9 \mathrm{GHz}$. But such value can reach $39 \mathrm{GHz}$ if the filter order is set to be 7 th order. Fig. $3 b$ shows the effective phase transfer function of 10 filters concatenation. The result is similar to the individual situation. However, compared with fig. $2 \mathrm{~b}$ it can be seen that the linear region becomes much smaller and the frequency dependency becomes more severe in all orders.

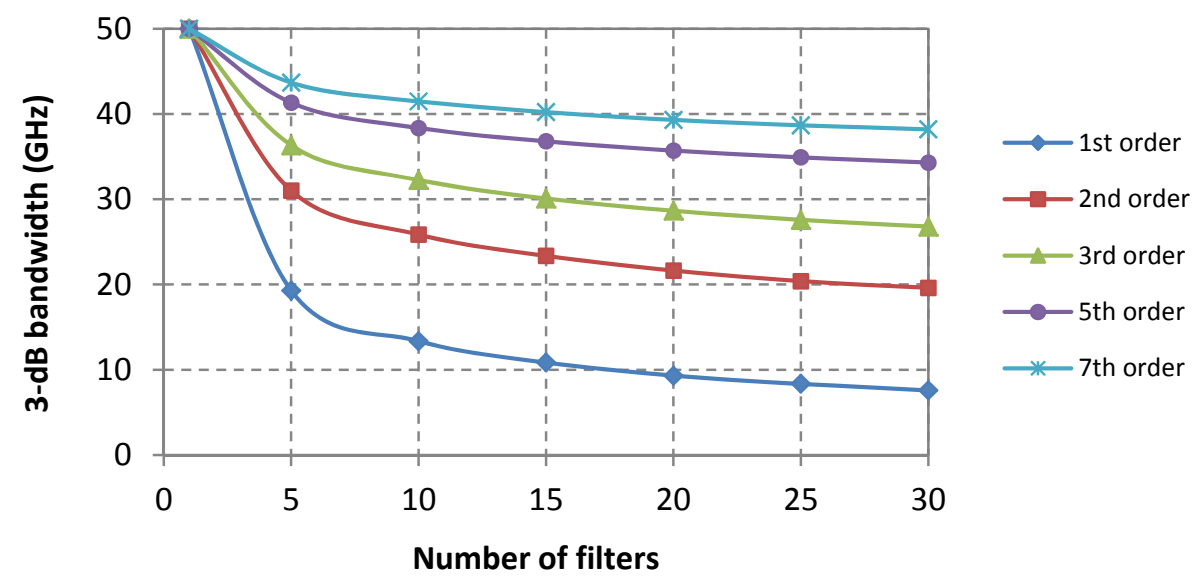

Figure 3a. 3-dB bandwidth changing with filters concatenation in different orders

Transmission Phase after 10 Filters Concatenation

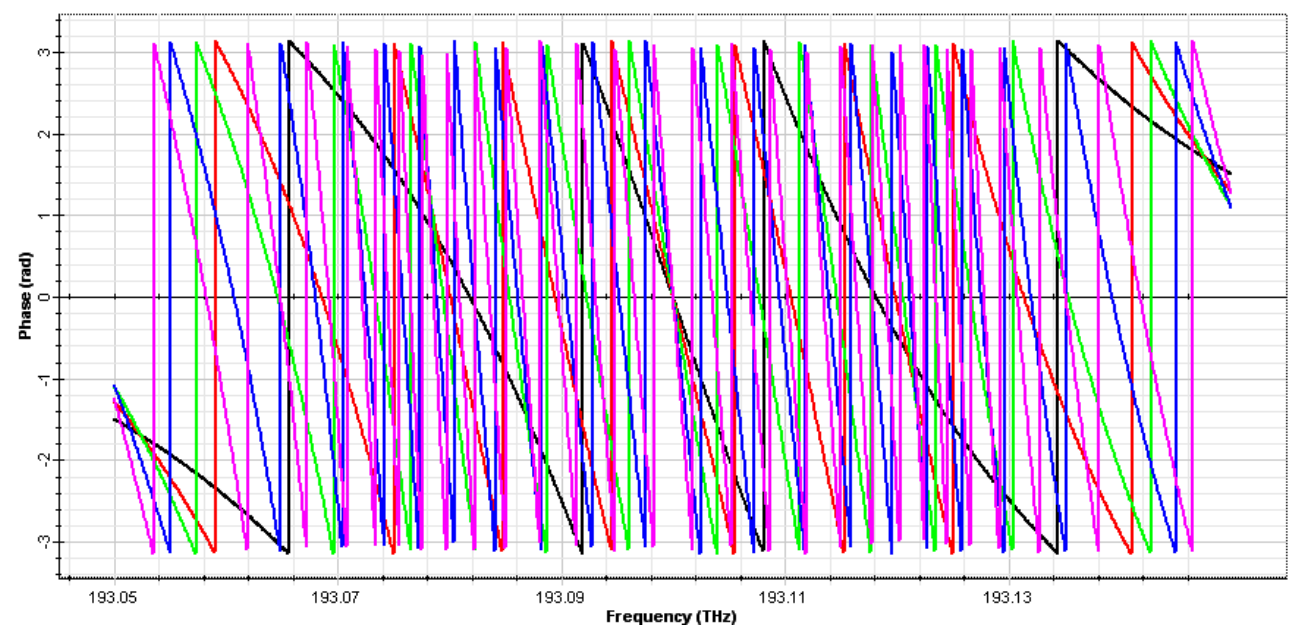

Figure 3b. Phase transfer function of different orders after 10 cascaded Butterworth filters 


\subsection{Bessel, FBG and F-P filters}

Although Butterworth filter is one of the most important filters, there are several other types of filter models, such as Bessel, FBG and F-P, which can also be used in the real condition. In this part we will study such these three kinds of filters and compared their performance with the Butterworth filter. The transfer functions of Bessel filter, FBG and F-P can be described as the following:

- Bessel transfer function [13]:

$$
\begin{gathered}
H(s)=\alpha \frac{d_{0}}{B_{n}(s)} \\
s=j\left(\frac{2\left(f-f_{c}\right) \cdot w_{b}}{B}\right)
\end{gathered}
$$

where $B_{n}$ is an $\mathrm{n}^{\text {th }}$-order Bessel polynomial, $B_{n}=\sum_{k=0}^{n} d_{k} s^{k} ; d_{0}$ is a normalizing constant, $d_{0}=\frac{(2 n) !}{2^{n} \cdot n !}, w_{b}$ denotes the normalized $-3 \mathrm{~dB}$ bandwidth.

- For the FBG, its reflectivity spectrum servers the role of the transfer function [14]:

$$
H(\delta)=\frac{i k \sin (q L)}{q \cos (q L)-i \delta \sin (q L)}
$$

where $\delta$ is a measure of detuning from the Bragg frequency and can be defined as $\delta(\omega)=(\bar{n} / c)\left(\omega-\omega_{B}\right), \bar{n}$ is the refractive index of the fiber mode, $k$ is the coupling coefficient, $L$ is the length of grating, $q$ is to be determined and obey the dispersion relation of $q= \pm \sqrt{\delta^{2}-k^{2}}$

- F-P transfer function [13]:

$$
\begin{array}{r}
H(f)=\alpha \frac{1-R}{1-R^{*} \exp \left(2 \pi J \frac{\left(f-f_{C}\right)}{F S R}\right)} \\
R=\frac{2+\left(\frac{\pi B}{F S R}\right)^{2}-\sqrt{\left(2+\frac{\pi B}{F S R}\right)^{2}-4}}{2}
\end{array}
$$

where FSR is the free spectral range and $B$ is the effective bandwidth. The amplitude and phase transfer functions of Butterworth, Bessel, FBG and F-P filters are shown in fig. 4a and fig. 4b, respectively. From fig. 4 we can see that the F$\mathrm{P}$ filter has the flattest transmission spectrum and it also has large phase linear region. Combining these two factors it can be known that the F-P filter has less effect to the signal in the individual condition. However, since F-P filter has smallest flat top region in the transmission spectrum, it decides that the effective bandwidth will decrease seriously with the cascaded number increasing. This can be seen in fig. 5. The size of flat top region of Bessel filter is a little larger than F-P filter and it also has phase linear region as large as F-P filter. So it can be derived that the effective bandwidth of cascaded Bessel filters is larger than that of the F-P filters in the same condition, as shown in fig. 5. Besides, we can also derive that the performance of cascaded Bessel filters is better than that of F-P filters. This can be demonstrated in part 4 (fig. 7 and fig. 8). Fig. 4 a also shows that the Butterworth filter and FBG have wide flat top region. This makes the effective bandwidth decreases slowly when several filters are cascaded. However, compared with Bessel and F-P filter, the phase linear region of the Butterworth filter and FBG is narrower. This will take more effect to the signal in the cascaded condition.

Fig. 5 shows the variation of effective bandwidth with the cascaded filters in the situation of center frequency aligned and misaligned. When center frequency of all cascaded filters is not aligned, it will make the effective bandwidth much narrower. From fig. 5 we can see that whatever the center frequency of all cascaded filters aligned or not, the Butterworth and the FBG have much wider bandwidth than F-P and Bessel after several filters concatenation. In the situation of center frequency aligned, after 20 filters concatenation the effective bandwidth of Butterworth and FBG is 
about $30 \mathrm{GHz}$ and about $10 \mathrm{GHz}$ for the F-P and Bessel. Through the comparison between the center frequency alignment and misalignment ( $\pm 5 \mathrm{GHz}$ deviation) we can also see that with center frequency alignment, 3 more filters can be cascaded to get the same bandwidth.

Combining Fig. 4a and Fig. 5, we can make a conclusion that when the amplitude transfer function of a filter is much closer to the ideal rectangle transfer function, the effective bandwidth of cascaded filters is much wider. This can be explained through the normalized multiplication of transfer function. After many filters concatenation, the effective transfer function is very close to the rectangle transfer function and the effective bandwidth is very close to the width of flat top region. So if the width of flat top region is much closer to the bandwidth of a filter, it will reduce the effect to the effective bandwidth after such filters concatenation.

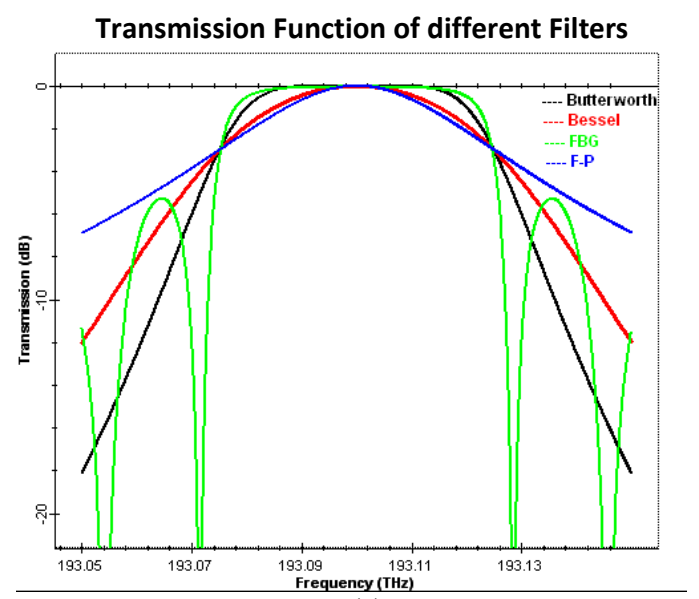

(a)

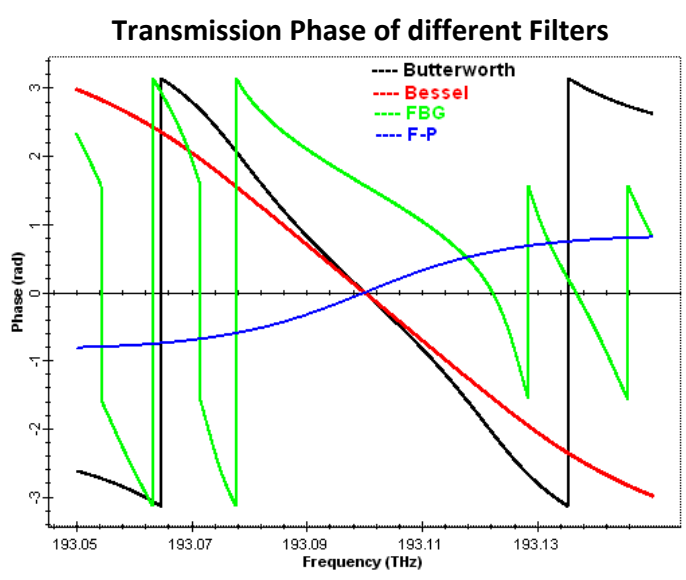

(b)

Figure 4. (a) Amplitude transfer function of four kinds of filters; (b) phase transfer function of four kinds of filters

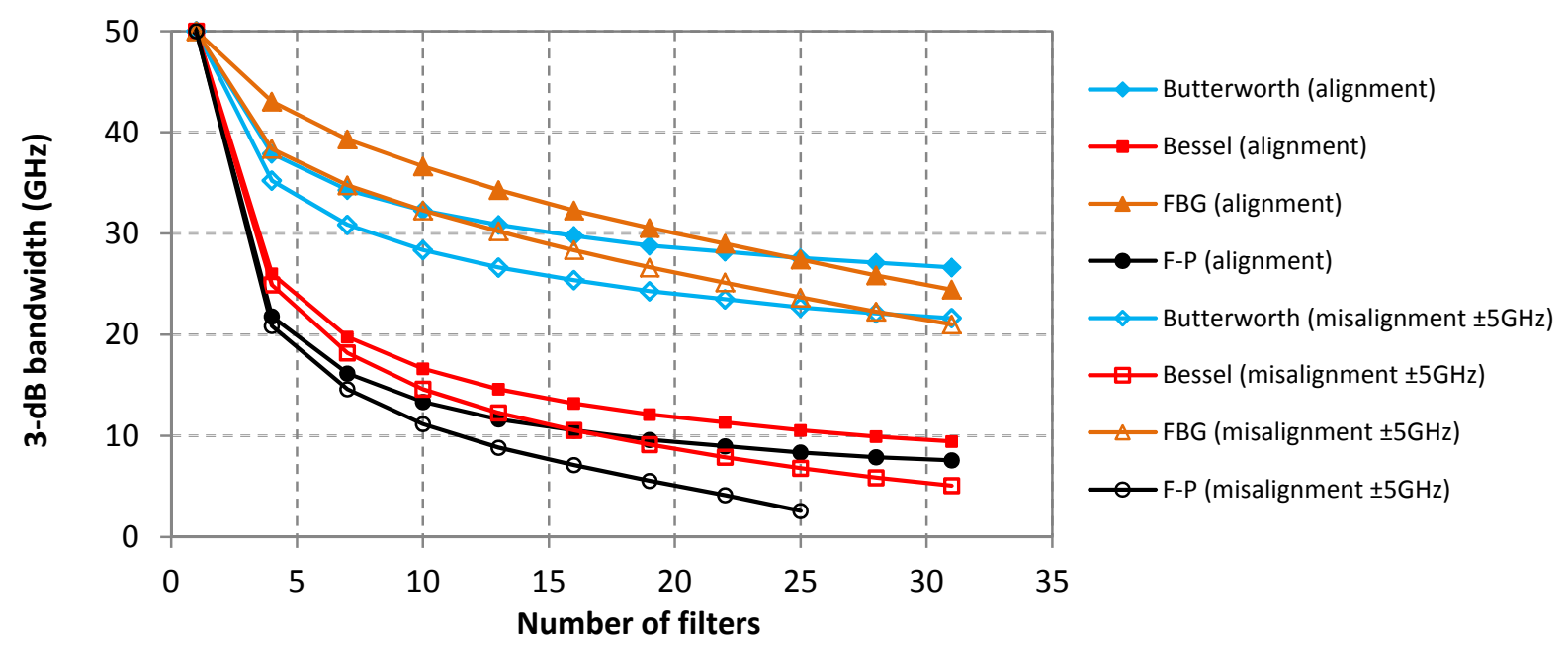

Figure 5. 3-dB bandwidth as a function of number of filters for different kinds of filters 


\section{SIMULATION SETUP}

In order to make the simulation structure much simple and close to the real condition, we design a simulation scheme as shown in fig. 6. The modulation formats used in the simulation are 40 Gbps NRZ-DQPSK and 100 Gbps PM-NRZDQPSK, respectively. The loop controller is used to decide how many times the signal passes through the filter. In the scheme the Erbium-doped fiber amplifiers (EDFAs) are used to compensate the insertion loss and attenuation introduced by single mode fiber (SMF) and dispersion compensating fiber (DCF). The DCF is used to compensate the accumulated dispersion generated by the SMF. In order to avoid introducing nonlinear effects, the power laughed into the DCF is kept lower than $-4 \mathrm{dBm}$. Then the signal goes into a 10: 90 power splitter to divide into two beams. One goes to the optical spectrum analyzer to observe the signal spectrum. The other one goes to the receiver. Finally a bit error rate (BER) analyzer is connected to the receiver to analyze the performance of the received signal. Since the input power of both kinds of fibers is lower than $-4 \mathrm{dBm}$, we don't consider the nonlinear effect in the simulation. For making the simulation results much close to the real condition, all parameters of each component in the simulation is based on commercial components. The simulation parameters are indicated in Table 1.

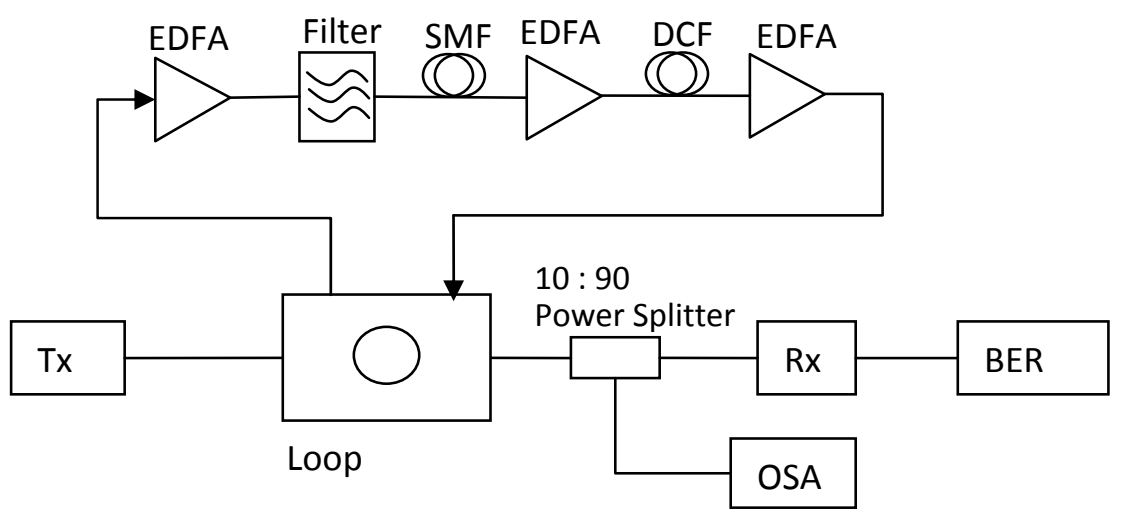

Figure 6. Block diagram of back-to-back transmission scheme. Tx: (NRZ-DQPSK or PM-NRZ-DPQSK) Transmitter; Rx: (NRZ-DQPSK or PM-NRZ-DPQSK) Receiver; OSA: Optical Spectrum Analyzer; BER Analyzer: Bit Error Rate Analyzer; EDFA: Erbium-doped fiber amplifier; SMF: single mode fiber; DCF: dispersion compensation fiber.

Table 1. Simulation parameters

\begin{tabular}{|c|c|c|c|}
\hline Name & Parameter & Unit & Value \\
\hline $\begin{array}{c}\text { Filters (Butterworth, } \\
\text { Bessel, FBG and F-P) }\end{array}$ & Insertion loss & $\mathrm{dB}$ & 5 \\
\hline EDFA1 & Gain & $\mathrm{dB}$ & 10 \\
\hline EDFA2 & Gain & $\mathrm{dB}$ & 15 \\
\hline EDFA3 & Gain & $\mathrm{dB}$ & 15 \\
\hline \multirow{5}{*}{ SMF } & Noise Figure (all amplifiers) & $\mathrm{dB}$ & 5 \\
\hline & PMD & $\mathrm{ps} / \mathrm{km}$ & 0.06 \\
\cline { 2 - 4 } & Chromatic dispersion & $\mathrm{ps} / \mathrm{nm} \cdot \mathrm{km}$ & 16.0 \\
\cline { 2 - 4 } & Dispersion slope & $\mathrm{ps} / \mathrm{nm} 2 \cdot \mathrm{km}$ & 0.075 \\
\cline { 2 - 4 } & Attenuation & $\mathrm{dB} / \mathrm{km}$ & 0.2 \\
\cline { 2 - 4 } & Length & $\mathrm{km}$ & 90 \\
\hline \multirow{5}{*}{ DCF } & PMD & $\mathrm{ps} / \mathrm{km}$ & 0.05 \\
\cline { 2 - 4 } & Chromatic dispersion & $\mathrm{ps} / \mathrm{nm}$ & -48 \\
\cline { 2 - 4 } & Dispersion slope & $\mathrm{ps} / \mathrm{nm}$ & -0.15 \\
\cline { 2 - 4 } & Attenuation & $\mathrm{dB} / \mathrm{km}$ & 0.26 \\
\cline { 2 - 4 } & Length & $\mathrm{km}$ & 30 \\
\hline
\end{tabular}


For analyzing the signal impairment from EOP, we first need to understand the definitions of eye opening. As mentioned in [12], eye opening can be defined as

$$
\text { Eye Opening }=V_{1}-V_{0}
$$

Where $V_{l}$ is the voltage level of the minimum " 1 " rail at the eye center, and $V_{0}$ is the voltage level of the maximum " 0 " rail. So the EOP can be described as

$$
E O P=10 \log \left(\frac{V_{u, 1}-V_{u, 0}}{V_{d, 1}-V_{d, 0}}\right)
$$

where the subscript " $u$ " represents the undistorted state of the signal and the subscript " $d$ " represents the distorted state of the signal.

\section{ANALYSIS OF SIMULATION RESULTS}

Simulations are done with 40 Gbps NRZ-DQPSK and 100 Gbps PM-NRZ-DQPSK formats. The simulation results with both situations of filter's center frequency aligned and misaligned are presented in Fig. 7 and Fig. 8 . Fig. 7 shows that in the situation of filters' center frequency aligned, the Butterworth filter and FBG show the best performance while the FP shows the worst performance. With 1dB EOP, it is possible to make $18 \mathrm{FBG}$ and Butterworth filters cascaded, but only 9 for the F-P. Fig. 8 shows that with center frequency misaligned arbitrarily of $\pm 5 \mathrm{GHz}$, the influence to the Butterworth filter and FBG is the biggest. In such situation, only about 12 Butterworth filters and FBG can be cascaded. This value is similar to that of Bessel, which means that the center frequency misalignment of $\pm 5 \mathrm{GHz}$ affects the Bessel filter less.

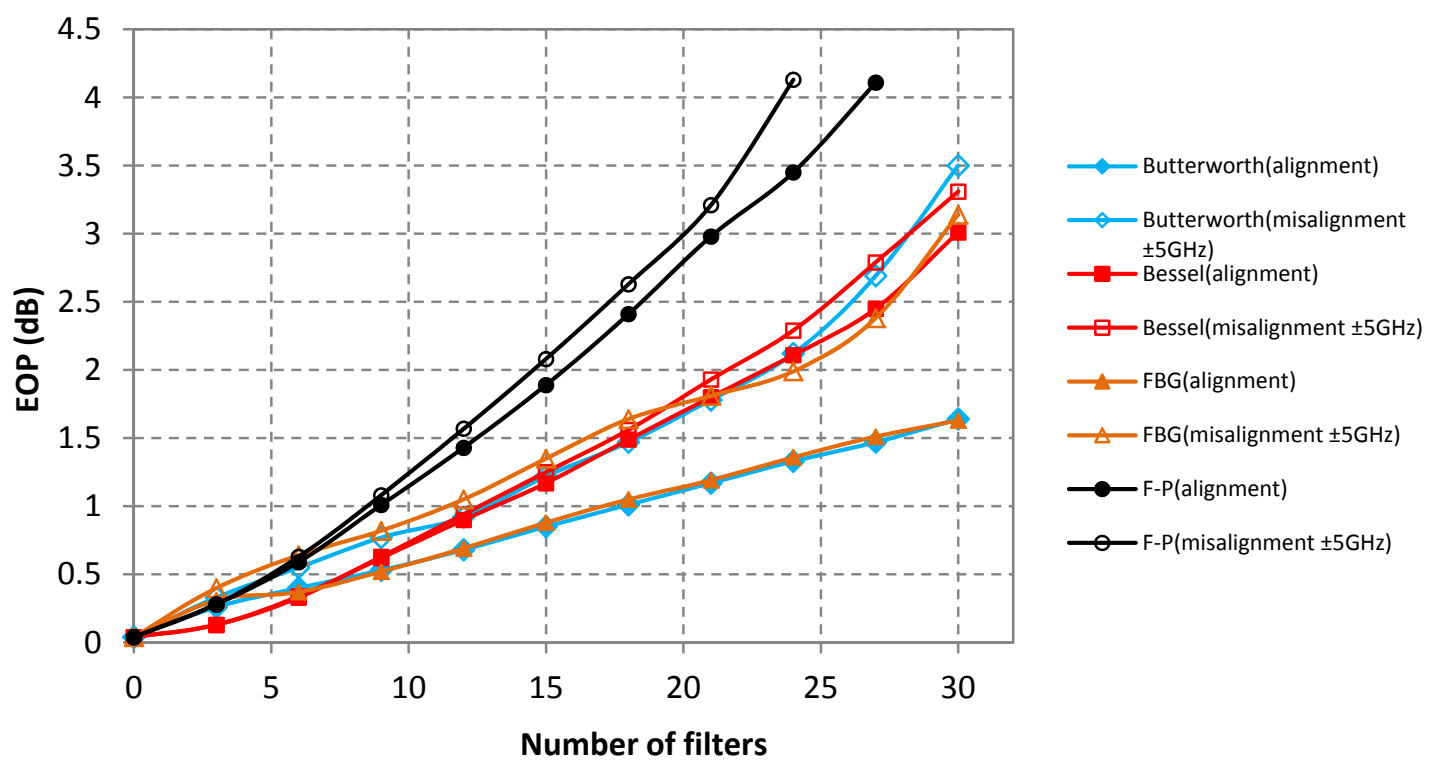

Figure 7. EOP as a function of number of filters in the 40 Gbps NRZ-DQPSK system with (a) all the filters' center frequency aligned and (b) filters' center frequency misaligned of $\pm 5 \mathrm{GHz}$ 


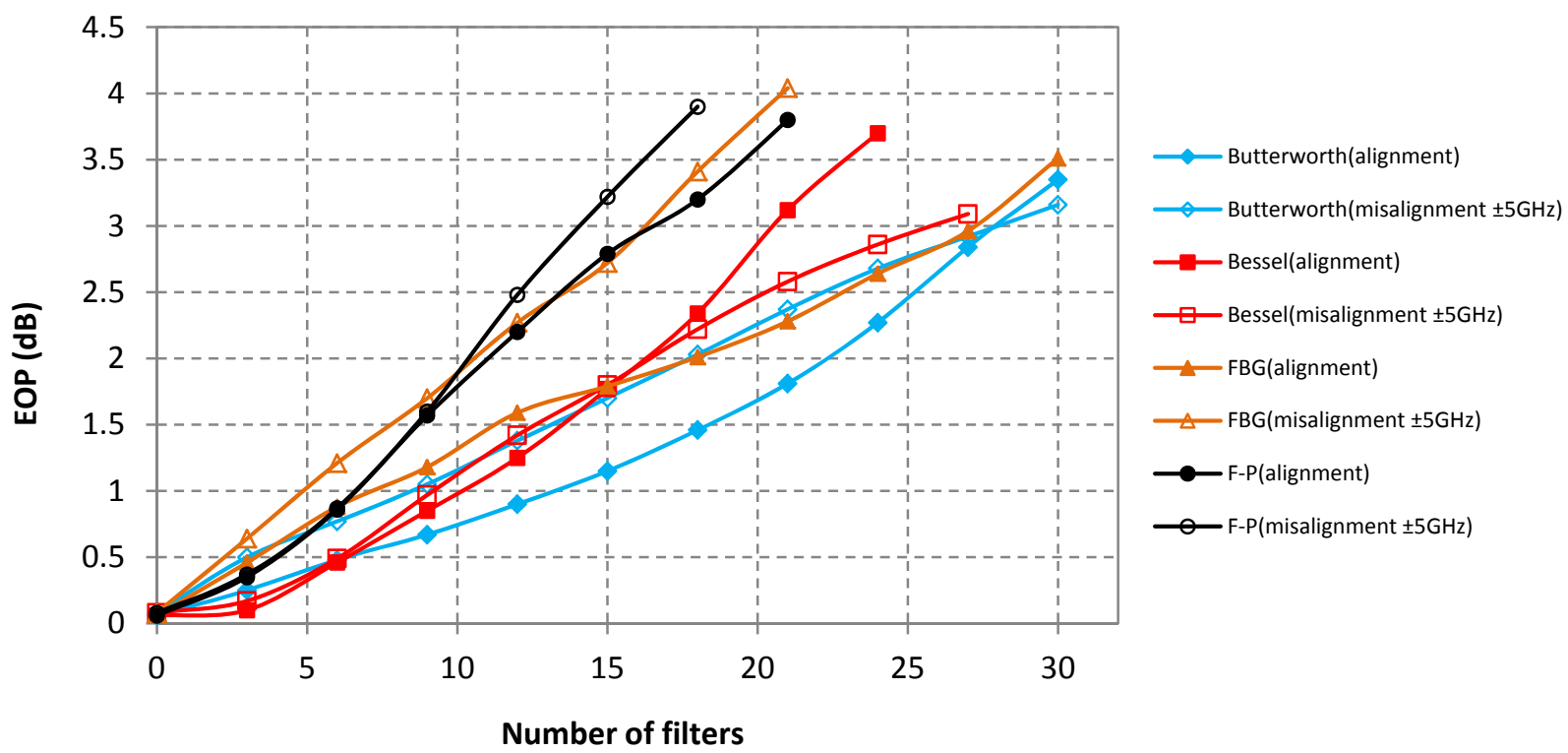

Figure 8. EOP as a function of number of filters in the 100 Gbps PM-NRZ-DQPSK system with (a) all the filters' center frequency aligned and (b) filters' center frequency misaligned of $\pm 5 \mathrm{GHz}$

In the 100 Gbps PM-NRZ-DQPSK transmission system, when all the filters' center frequency aligned, the Butterworth filter shows the best performance as in 40 Gbps DQPSK system and about 12 filters can be cascaded with $1 \mathrm{~dB}$ EOP, as shown in fig. 8. For the other three kinds of filters, the cascaded number is 6,6 and 10 , corresponding to FBG, F-P and Bessel, respectively. In the situation of filters' center frequency misaligned, signal impairment caused by FBG and Butterworth concatenation becomes more serious. Only 5 FBG and 8 Butterworth filters can be cascaded within a $1 \mathrm{~dB}$ EOP. But the Bessel shows a better performance as in a 40 Gbps DQPSK system. In the limited range of 1dB EOP, 9 Bessel filters can be cascaded. The performance of cascaded F-P is similar in two conditions. From Fig. 7 and Fig. 8 we can see that whatever in 40Gpbs DQPSK system or 100Gpbs PM-DQPSK system, signal impairment caused by both F-P and FBG with center frequency misalignment of $\pm 5 \mathrm{GHz}$ is very small.

Since Butterworth model is one of the most important filter models and most of the filters can work as a 3rd Butterworth filter [2], it is significant to study the signal impairment caused by different orders of Butterworth model. Fig. 3 shows that with the order increasing, the effective bandwidth of filters concatenation is wider. In other words, that means the higher-order filters concatenation do less effect of signal spectrum clipping than that caused by the lower-order filter concatenation. However, Fig. 2b shows that the transmission phase depends seriously on the frequency with the order increasing. Therefore, although it allows more higher-order filters to be cascaded to get a permitted effective bandwidth, it does not mean that signal impairment will be mitigated. The signal impairment caused by cascaded filters is decided by both transmission amplitude and transmission phase, which can be seen from the simulation results. Fig. 9 shows the signal impairment caused by different order Butterworth filters. Combining Fig. 3 and Fig. 9 we can see that although the effective bandwidth of cascaded $5^{\text {th }}$ or $7^{\text {th }}$ order Butterworth filters is wider that of cascaded $3^{\text {rd }}$ order Butterworth filters, they take more impairment to the signal.

We can also see that the signal impairment caused by the cascaded $1^{\text {st }}$ order Butterworth filters is the worst among all the orders. This is due to the serious decrease of the effective bandwidth. It can be concluded that property of cascaded filters is determined by the effective bandwidth and the transmission phase. In the low-order situation, such as 1st and $2^{\text {nd }}$, the effective bandwidth takes the dominant role. That is why we can see that the cascaded $2^{\text {nd }}$ order Butterworth filters introduces less impairment to the signal than the $1^{\text {st }}$ order. But in high-order situation, which is higher than $3^{\text {rd }}$ order (including $3^{\text {rd }}$ order), the transmission phase takes the dominant role in the property. That is why the cascaded $5^{\text {th }}$ and $7^{\text {th }}$ order Butterworth filters take more impairment to the signal than the 3 rd order. The $2^{\text {nd }}$ and $3^{\text {rd }}$ order Butterworth 
filters have better performance than other orders. However, if we just make a comparison between the $2^{\text {nd }}$ and $3^{\text {rd }}$ order, we find that with $1 \mathrm{~dB}$ EOP, 10 more filters can be cascaded in a 40 Gbps DQPSK system and 5 more can be cascaded in a $100 \mathrm{Gbps}$ PM-DQPSK system when we use the $2^{\text {nd }}$ order Butterworth filter. The effect of signal spectrum clipping caused by filters concatenation can be seen in fig. 10. And the eye diagrams of the signal distortion are shown in fig. 11.

Through comparison between fig. 7 and fig. 9(a) or fig. 8 and fig. 9(b), we can see that the F-P filter property is almost the same as the first order Butterworth filter model. This is because in the real condition the F-P filter can be approximately to the first order Butterworth filter model [15].

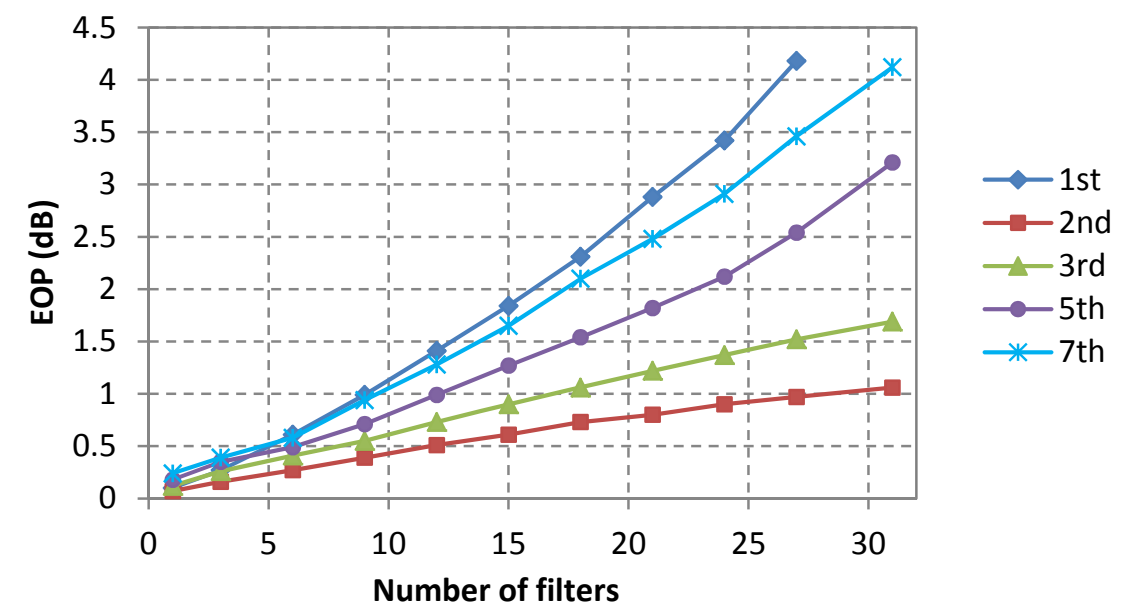

(a)

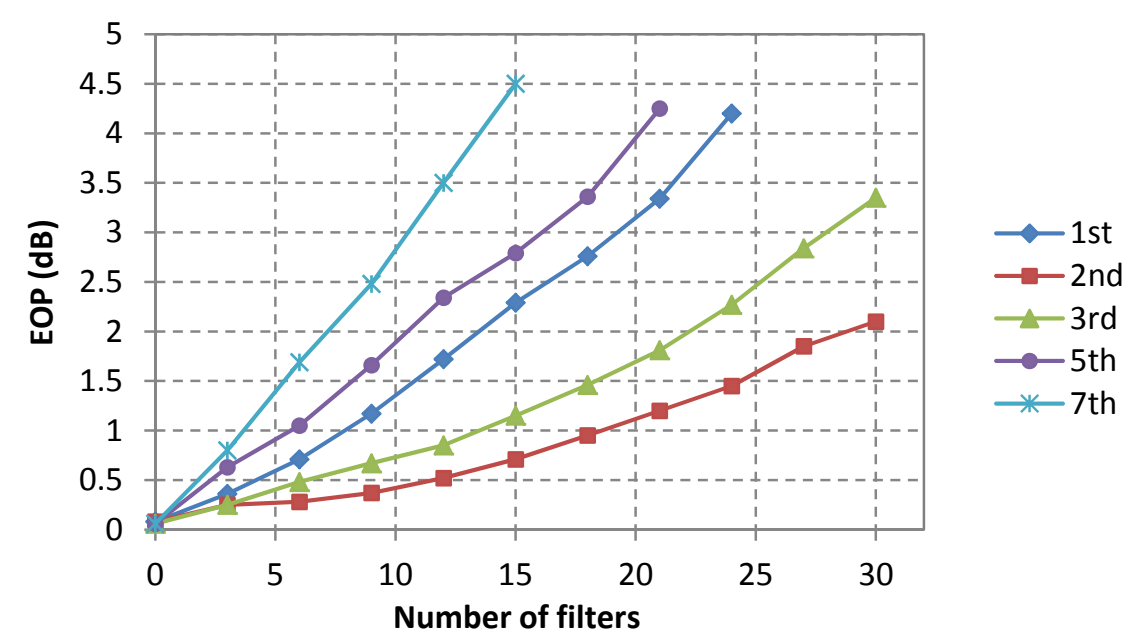

(b)

Figure 9. EOP as a function of number of filters with different orders of Butterworth model in (a) a 40 Gbps NRZ-DQPSK system and (b) a 100 Gbps PM-NRZ-DQPSK system 


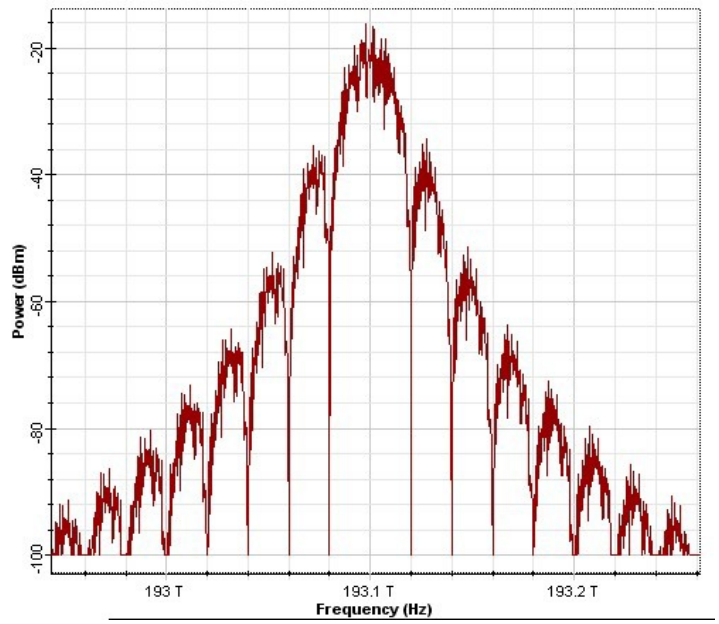

(a)

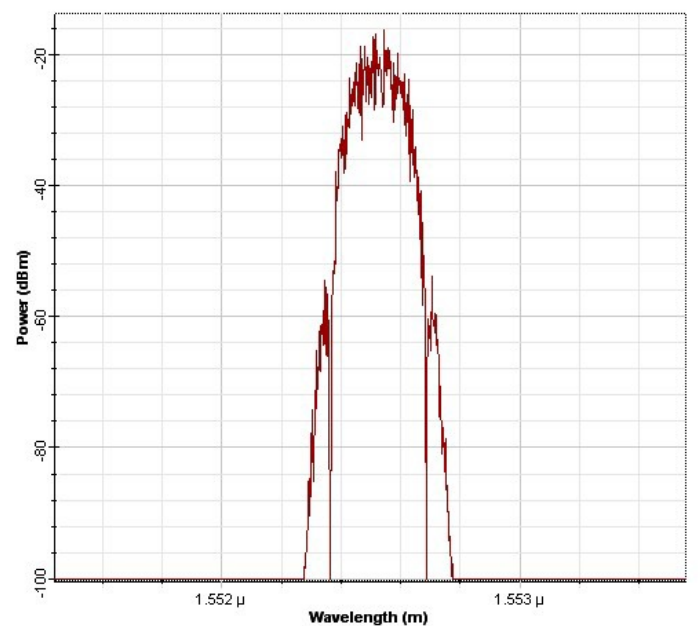

(b)

Figure 10. Signal Spectrum (a) after 13 rd order Butterworth filter; (b) after 18 3rd order Butterworth filters

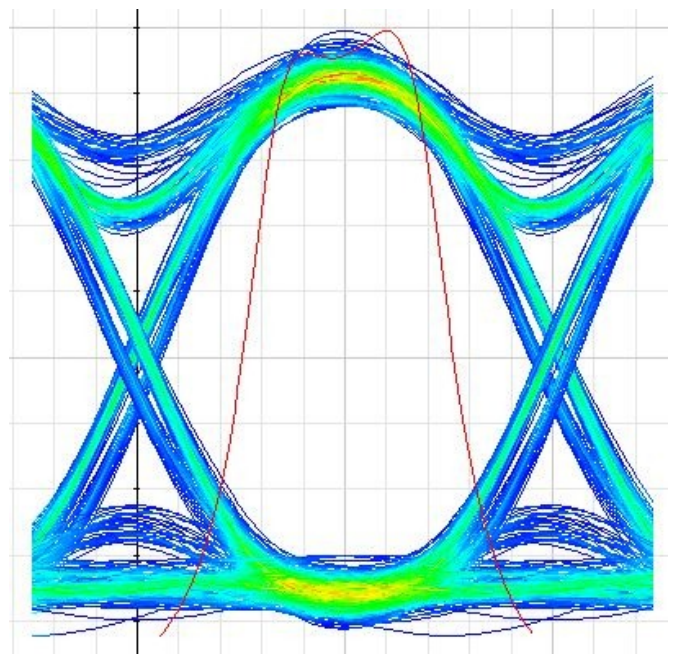

(a)

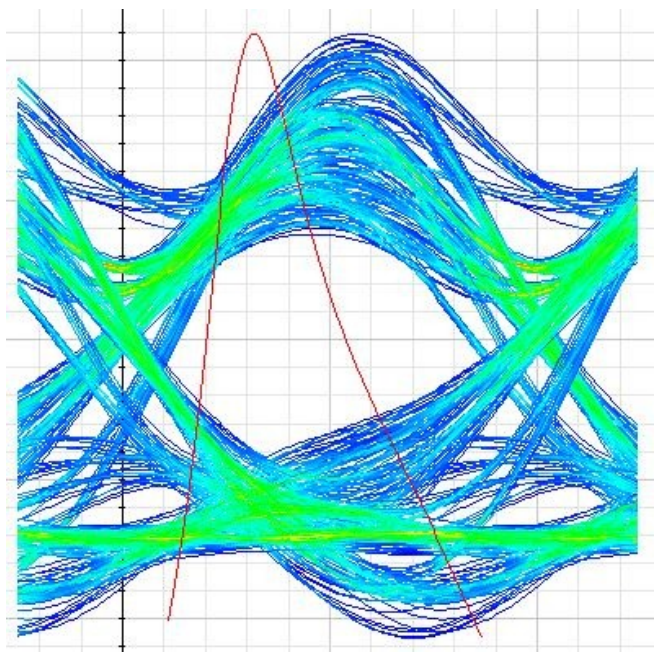

(b)

Figure 11. Eye diagrams, (a) after 1 3rd order Butterworth filter; (b) after 18 3rd order Butterworth filters concatenation.

\section{CONCLUSION}

In this paper we study and simulate the signal impairment caused by different kinds of filters. The theoretical analysis and simulation results show that the Butterworth filter has the best performance among those four kinds of filters. In the 40 Gbps DQPSK system, about 18 Butterworth filters can be can cascaded with 1dB EOP in the situation of all filters' center frequency aligned and 12 in that of filters' frequency misaligned of $\pm 5 \mathrm{GHz}$. In the $100 \mathrm{Gbps}$ PM-DQPSK system, the numbers of cascaded Butterworth filters are 12 and 9 for both alignment and misalignment condition, respectively. Besides, study and simulation shows that the $2^{\text {nd }}$ Butterworth has the best performance. If in the real condition a filter can be made to be approximately to the $2^{\text {nd }}$ Butterworth model, about 10 more filters can be cascaded in 40 Gbps DQPSK systems and 5 more in 100 Gbps PM-DQPSK systems, with -1dB EOP. 


\section{ACKNOWLEDGMENTS}

The authors would like to acknowledge support from the China Scholarship Council (CSC).

\section{REFERENCES}

[1] Stelios Sygletos, Anna Tzanakaki and Ioannis Tomkos, "Numerical Study of Cascadability Performance of continuous Spectrum Wavelength Blocker/Selective Switch at 10/40/160 Gb/s," IEEE Photonics Technology Letters, 18(24), 2608-2610 (2006)

[2] John D. Downie, Ioannis Tomkos, Neophytos Antoniades and Aleksandra Boskovic, "Effects of Filter Concatenation for Directly Modulated Transmission Lasers at 2.5 and $10 \mathrm{~Gb} / \mathrm{s}$," J. Lightwave Technology, 20(2), 218-227 (2002)

[3] Y. Tang and W. Shieh, "Filter concatenation impact on $107-\mathrm{Gb} / \mathrm{s}$ coherent optical OFDM system," in proc. OECC, FQ2, 1-2 (2009)

[4] Elias Giacounidis, Ioannis Tomkos, and J. M. Tang, "Adaptive Modulation-Induced Reduction in Filter Concatenation Impairment for Optical OFDM Metro/Regional Systems," J. Opt. Commun. Netw., 3(7), 587-593 (2011)

[5] Daniel M. Pataca, Julio C. R. F. Oliveira, Antonio A. Juriollo and Adolfo F. Herbster, "Transmission of a $20 \mathrm{~Gb} / \mathrm{s}$ NRZ OOK Signal Throughout a $390 \mathrm{~km}$ Fiber Link and a Cascade of 10 x $50 \mathrm{GHz}$ Filters and 9 x EDFAs," J. Microwaves Optoelectronics and Electromagnetic Applications, 10(1), 143-154 (2011)

[6] Marcin Chochol, Josep M. Fabrega, Michela Svaluto Moreolo and Gabriel Junyent, "Optical Filter Cascading Effects in a Phase Modulated Coherent Optical OFDM Transmission System Based on Hartley Transform," in proc. ICTON, Tu.P.3, 1-4 (2012)

[7] Sorin Tibuleac and Mark Filer, "Transmission Impairments in DWDM Networks with Reconfigurable Optical AddDrop Multiplexers,” J. lightwave Technology, 28(4), 557-568 (2010)

[8] John D. Downie and A. Boh Ruffin, "Analysis of Signal Distortion and Crosstalk Penalties Induced by Optical Filters in Optical Networks," J. Lightwave Technology, 21(9), 1176-1186 (2003)

[9] Xiang Liu, Xing Wei, A. H. Gnauck. C. R. Doerr and S. Chandrasekhar, "Analysis of Loss Ripple and Its Application to the Mitigation of Optical Filtering Penalty," IEEE Photonics Technology Letters, 17(1), 82-84 (2005)

[10] Helene Chotar, Yves Painchaud, Alain Mailloux, Michel Morin, Francois Trepanier and Martin Guy, "Group delay Ripple of Cascaded Bragg Grating Gain Flattening Filters," IEEE Photonics Technology Letters, 14(8), 1130-1132 (2002)

[11] G. Lenz, B. J. Eggleton, C. K. Madsen, C. R. Giles and G. Nykolak, "Optimal Dispersion of Optical Filters for WDM Systems," IEEE photonics Technology Letters, 10(4), 567-569 (1998)

[12] John D. Downie, "Relationship of Q Penalty to Eye-Closure Penalty for NRZ and RZ Signals With SignalDependent Noise," J. Lightwave Technology, 23(6), 2031-2038 (2005)

[13] Optiwave, [OptiSystem Component Library: Optical Communication System Design Software, Version 11.0], Optiwave, Ottawa, 847-848 (2012)

[14] Govind P. Agrawal, [Lightwave Technology: Components and Devices], John Wiley \& Sons, New Jersey, 62-69 (2004)

[15]N. N. Khrais, A. F. Elrefaie, R. E. Wagner and S. Ahmed, "Performance of Cascaded Misaligned Optical (De)Multiplexers in Multiwavelength Optical Networks," IEEE Photonics Technology Letters, 8(8), 1073-1075 (1996)

[16]E. Forestieri and G. Prati, "Narrow Filtered DPSK Implements Order-1 CAPS Optical Line Coding," IEEE Photonics Technology Letters, 16(2), 662-664 (2004) 